\title{
Combining a scanning near-field optical microscope with a picosecond streak camera: Statistical analysis of exciton kinetics in GaAs single-quantum wells
}

\author{
U. Neuberth, L. Walter, G. von Freymann, B. Dal Don, H. Kalt, and M. Wegener ${ }^{\mathrm{a})}$ \\ Institut für Angewandte Physik, Universität Karlsruhe (TH), D-76131 Karlsruhe, Germany \\ G. Khitrova and H. M. Gibbs \\ Optical Sciences Center, University of Arizona, Tucson, Arizona 85721
}

(Received 17 January 2002; accepted for publication 21 March 2002)

\begin{abstract}
Combining a low-temperature scanning near-field optical microscope with a picosecond streak camera allows us to measure the complete wavelength-time behavior at one spot on the sample within about $13 \mathrm{~min}$ at excitation powers of $100 \mathrm{nW}$. We use this instrument to measure the variation of relaxation times in disordered single-GaAs quantum wells with sample position. (C) 2002 American Institute of Physics. [DOI: 10.1063/1.1477274]
\end{abstract}

Soon after the invention of scanning probe microscopy, many researchers got excited about combining scanning probe techniques with the established methods of femto- or picosecond optical spectroscopy—a combination, which would open the door to a whole new family of experiments. Several pioneering experiments were performed indeed, ${ }^{1-4}$ however, much of the enthusiasm was largely damped over the years because of enormous technical difficulties which inhibited a routine application of these techniques. Here, we combine a low-temperature scanning near-field optical microscope (using uncoated optical fiber tips, allowing for large throughput) with a picosecond streak camera. While this combination has only modest spatial [200-300 nm resolution at $800 \mathrm{~nm}$ photoluminescence (PL) wavelength, see Fig. 1] and temporal $(2-10 \mathrm{ps})$ resolution, it does allow for routine operation. Compared to, e.g., using solid immersion lenses, our setup allows for more flexibility: Large field of view, coarse sample positioning up to $5 \mathrm{~mm}$ and ability to also work at around $400 \mathrm{~nm}$ wavelength. ${ }^{5,6}$

Figure 1 depicts the instrument. The sample is cooled by a variable-temperature He-flow cryostat with symmetric heat exchanger, while the fiber tip is held at room temperature. ${ }^{5-7}$ The scanning near-field optical microscope (SNOM) uses uncoated fiber tips, which are fabricated using a two-step selective etching process, ${ }^{8}$ leading to an apex radius around $20 \mathrm{~nm}$. The $150 \mathrm{fs}$ optical pulses at $\lambda_{\text {exc }}=700 \mathrm{~nm}$ wavelength ( $\Leftrightarrow \hbar \omega_{\text {exc }}=1.77 \mathrm{eV}$ photon energy) with an average power of $P_{\text {exc }}=100 \mathrm{nW}$ are sent into the single-mode optical fiber. We use the constant height mode ${ }^{8}$ with a typical tipsample separation of $100 \mathrm{~nm}$, i.e., the feedback loop is switched off. The excited PL of the sample is collected by the fiber tip and sent into a $0.5 \mathrm{~m}$ grating spectrometer connected to the streak camera. Without temporal resolution (i.e., without the streak camera), we typically detect several hundreds of counts per second in one wavelength channel (corresponding to $0.012 \mathrm{~nm}$ width) in the PL peaks under these conditions. As this number of counts is spreadout over time, i.e., over several hundreds of channels, one obviously

${ }^{a)}$ Electronic mail: martin.wegener@physik.uni-karlsruhe.de needs photon-counting capability and significant integration (exposure) times when introducing the streak camera. The time resolution is $2 \mathrm{ps}$ without spectrometer and $10 \mathrm{ps}$ for the actual experiments (e.g., Fig. 2).

The two samples investigated in this letter are highquality single-GaAs quantum wells (SQW). One is a $3.5 \mathrm{~nm}$ thin, $180 \mathrm{~s}$ growth-interrupted GaAs SQW with superlattice barriers [4 monolayers (ML) of AlAs, no interruption, $8 \mathrm{ML}$ of GaAs, 180 s growth interruption, etc.], grown by molecular-beam epitaxy on a (100) GaAs substrate. Accounting for an additional $10 \mathrm{~nm}$ GaAs cap layer, the SQW is 47 $\mathrm{nm}$ away from the sample surface (sample cat54). The other sample is a $5 \mathrm{~nm}$ thin GaAs SQW with 240 s growth interruption (sample cat83). For both samples, the excitation photon energy of $\hbar \omega_{\mathrm{exc}}=1.77 \mathrm{eV}$ is below the absorption edge of the superlattice barriers of the SQW. Further details of the samples can be found in Ref. 9.

Figure 2 shows a typical streak-camera image delivering the full spectral and temporal evolution of the PL at one spatial position of the fiber tip. As expected, one observes a series of sharp PL lines ${ }^{10,11}$ which reflect the localized exciton states within the atomically rough quantum well plane. Performing cuts at fixed photon energies through this data set allows one to extract the temporal dynamics versus photon

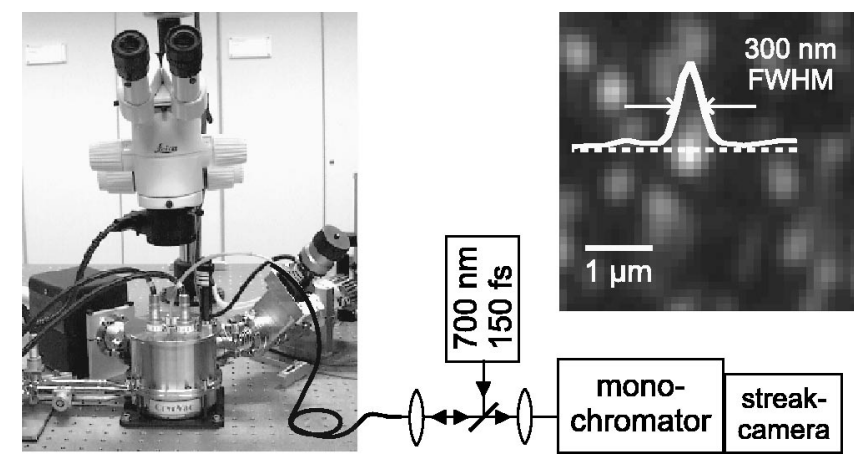

FIG. 1. Scheme of the experimental setup which combines a very compact low-temperature SNOM (see photograph) with a streak camera. A typical continuous wave PL image delivering features with $300 \mathrm{~nm}$ full width at half maximum resolution is depicted on the right-hand side. 


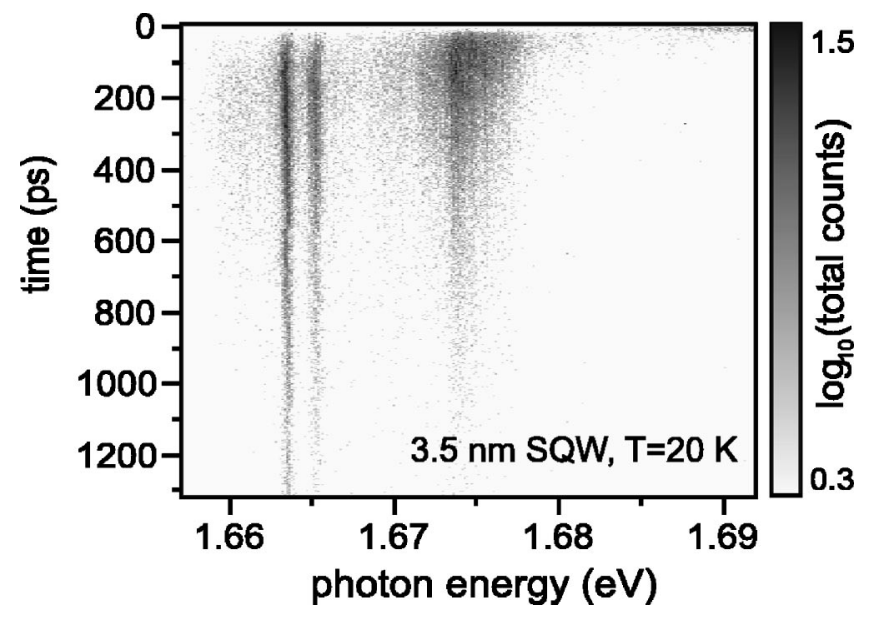

FIG. 2. Typical image of the streak camera at one position of the tip showing the PL intensity as a function of time and spectrometer photon energy. The intensity is given in total number of counts within 13 min exposure time. $P_{\text {exc }}=100 \mathrm{nW}$, sample (cat54) temperature is $T=20 \mathrm{~K}$.

energy which is depicted in Fig. 3. Here, the detection photon energy varies from 1.66 to $1.69 \mathrm{eV}$ in steps of $2 \mathrm{meV}$. One observes a rapid rise of the PL at large photon energies followed by a rapid decay, while PL rises and decays more slowly at small photon energies. Such overall behavior is well established from far-field time-resolved PL experiments on similar samples (see e.g., Ref. 12) and reflects the relaxation of excitons into the local potential minima of the disorder potential. However, in far-field PL experiments, one measures the spatially averaged PL dynamics while in our case, we can see how much the dynamics varies from one position of the disorder potential to another. Obviously, one gets an enormous wealth of information in this fashion. In

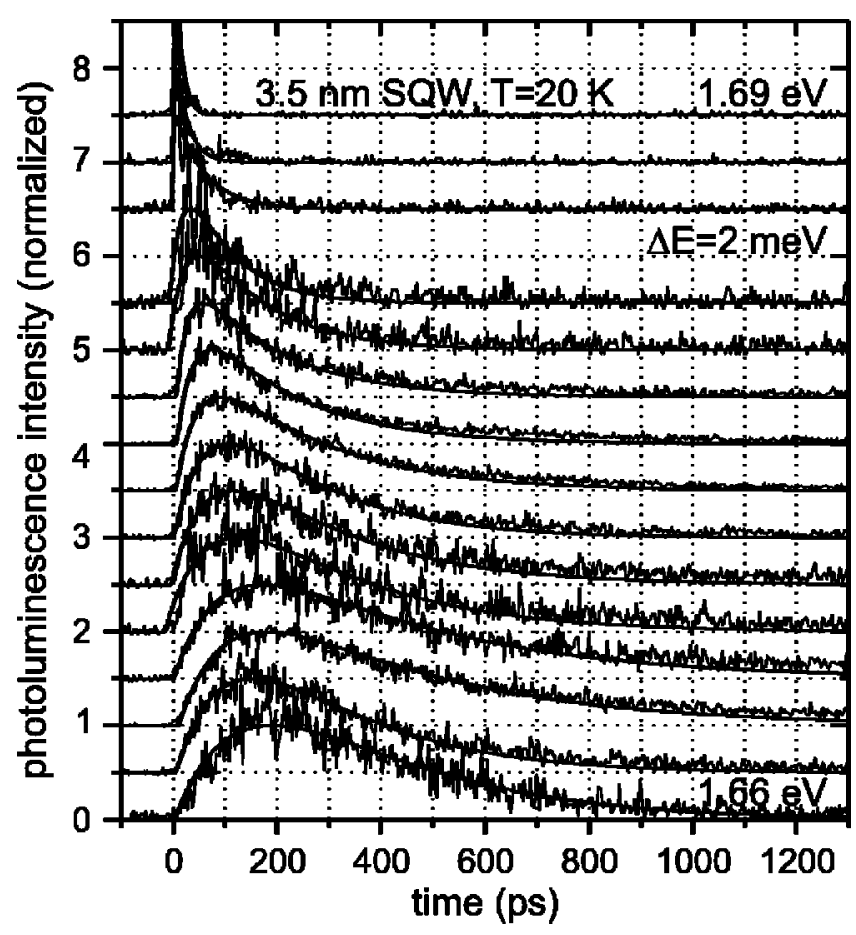

FIG. 3. Selected cuts (vertically displaced) through the data set shown in Fig. 2. Photon energy increases from $1.66 \mathrm{eV}$ (bottom) to $1.69 \mathrm{eV}$ (top) in 2 meV steps. The fits (full lines) match the experimental data (noisy curves) quite well. The time constants resulting from the fits are summarized in Figownloaded 06 Mar 2009 to 129.13.72.198. Redistribution subject

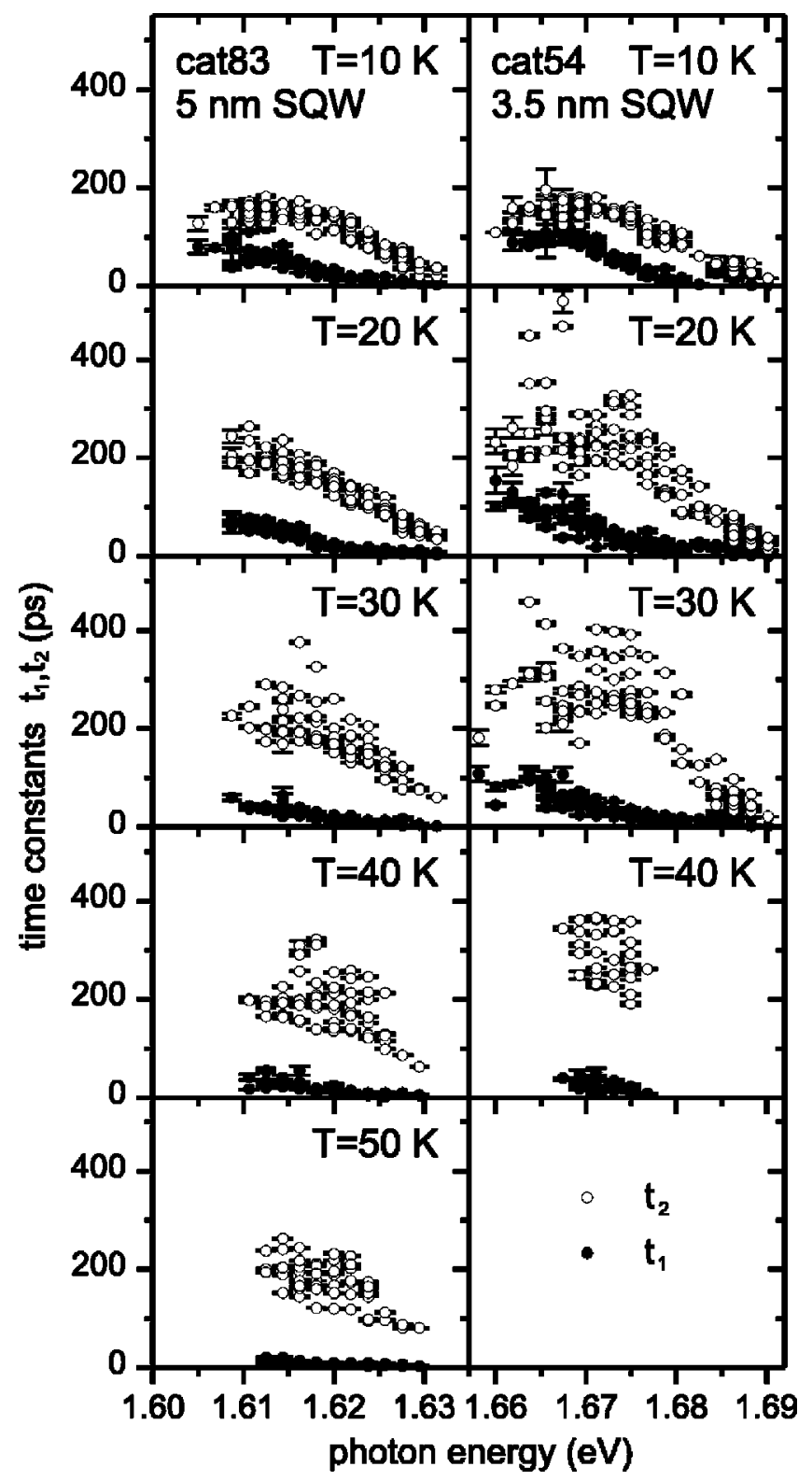

FIG. 4. Summary of results of fits to the data as, e.g., those shown in Fig. 3 and several others corresponding to tip positions on a grid with one micron pitch. The resulting time constants $t_{2}$ (open circles) and $t_{1}$ (full dots) are shown for two samples (cat83 and cat54) for sample temperatures increasing from 10 to $50 \mathrm{~K}$. The variation in time constants is due to the different spatial positions on the sample and not due to statistics (see error bars).

order to compress these data, we have performed fits to the PL intensity, $I(\hbar \omega, t)$, at fixed photon energy $\hbar \omega$ versus time $t$ using a simple fit formula (see e.g., Ref. 13)

$$
I(\hbar \omega, t)=I_{0}\left(\mathrm{e}^{-t / t_{2}}-\mathrm{e}^{-t / t_{1}}\right) .
$$

This procedure allows one to extract the times $t_{1}=t_{1}(\hbar \omega)$ and $t_{2}=t_{2}(\hbar \omega)>t_{1} \cdot t_{2}$ reflects the PL decay time and, if $t_{1}$ and $t_{2}$ are not too close, $t_{1}$ is the PL rise time. If $t_{1}$ and $t_{2}$ are equal, the concept of a rise time is actually not well defined.

Figure 4 summarizes two large sets of experiments at different positions on the samples cat 83 and cat54, respectively, for sample temperatures ranging from 10 to $50 \mathrm{~K}$ as indicated. Each point in this diagram stems from the fit to one PL trace such as the ones shown in Fig. 3. The variation of times at a given photon energy is not due to scatter in the data or due to uncertainties of the fits (see errors bars) but AIP license or copyright; see http://apl.aip.org/apl/copyright.jsp 
rather due to the actual variations of the times $t_{1}$ and $t_{2}$ from one position of the sample to another. At low temperatures and small photon energies, the times $t_{1}$ and $t_{2}$ are comparable, indicating that excitons are unable to acquire a thermal distribution within their lifetime. This is an important input for the interpretation of time-integrated experiments such as, ${ }^{7,9}$ which already suggested highly nonthermal distributions of excitons at $T=10 \mathrm{~K}$. Furthermore, the variation of the times from position to position on the sample is rather small at $T=10 \mathrm{~K}$. Both aspects gradually change toward higher temperatures: The decay time $t_{2}$ generally increases, giving excitons more time to relax into potential minima of the disorder potential (e.g., for sample cat 83 at $1.63 \mathrm{eV}$ photon energy from $t_{2}=40 \mathrm{ps}$ at $T=10 \mathrm{~K}$ to $t_{2}=80 \mathrm{ps}$ at $T$ $=50 \mathrm{~K})$. Theory ${ }^{14}$ suggests that this behavior is due to scattering of excitons with thermally activated acoustic phonons. Furthermore, the variation of relaxation times for both samples increases with increasing temperature (compare e.g., 10 and $30 \mathrm{~K}$ )—a behavior which has not been predicted by theory. A possible explanation is that at elevated temperatures, phonon absorption scatters and moves excitons within the disorder potential, leading to increased decay times $t_{2}$. The absorption of acoustic phonons might sensitively depend on the local environment, i.e., depend on whether the phonon energy fits to the local exciton wave functions and the associated eigenenergies. The latter are indeed expected to strongly vary from one position in the disorder potential to another.

Unfortunately, it is not possible to experimentally test whether the discussed behavior changes when further reducing the excitation power because of excessive integration times. In a simple estimate, the number of excitons, $N_{x}$, under the $(300 \mathrm{~nm})^{2}$ spot results from $N_{x}=E_{\text {exc }}[1$ $\left.-\exp \left(-\alpha L_{z}\right)\right] /\left(\hbar \omega_{\text {exc }}\right)$, with the energy of one laser pulse $E_{\text {exc }}=P_{\text {exc }} / 81 \mathrm{MHz}=1.23 \times 10^{-15} \mathrm{~J}$, the estimated absorption coefficient $\alpha=2 \times 10^{4} \mathrm{~cm}^{-1}$, the SQW thickness $L_{z}$ $=3.5 \mathrm{~nm}$ (for excitation below the superlattice barrier absorption) and $\hbar \omega_{\mathrm{exc}}=1.77 \mathrm{eV}$. This leads to a maximum number of excitons, directly after the laser pulse, of $N_{x}$ $=31$. This number is distributed over several tens of individual potential minima, and carrier transport (which we have neglected in the estimate) will further reduce this number. Thus, we expect slightly less than one exciton per potential minimum on the average for $P_{\text {exc }}=100 \mathrm{nW}$ excitation power.
In conclusion, we have measured the variation of relaxation times in disordered GaAs quantum wells by means of combining a low-temperature SNOM with a streak camera. The variation can be as large as a factor of two. It is interesting to compare our results with recent theoretical predictions of the distribution of radiative decay times of excitons as a function of their energy in the disorder potential. Reference 15 predicted a Porter-Thomas distribution of radiative decay rates for large exciton energies, i.e., many PL lines have small radiative decay rates and exponentially few have large decay rates. This is neither observed here nor in other work, ${ }^{16}$ which indicates that the kinetics of excitons in disorder potentials is still not fully understood theoretically.

The Karlsruhe team acknowledges support from the DFG and the BMBF, the Tucson group from NSF EPDT, JSOP (AFOSR and ARO), and NSA/ARO.

${ }^{1}$ J. B. Stark, U. Mohideen, and R. E. Slusher, Tech. Dig. Ser. Conf. Ed. 16, 82 (1995).

${ }^{2}$ S. Smith, N. C. R. Holme, B. Orr, R. Kopelman, and T. Norris, Ultramicroscopy 71, 213 (1998).

${ }^{3}$ S. Weiss, D. F. Ogletree, D. Botkin, M. Salmeron, and D. S. Chemla, Appl. Phys. Lett. 63, 2567 (1993).

${ }^{4}$ M. Achermann, U. Siegner, L.-E. Wernersson, and U. Keller, Appl. Phys. Lett. 77, 3370 (2000).

${ }^{5}$ G. von Freymann, D. Lüerßen, C. Rabenstein, M. Mikolaiczyk, H. Richter, H. Kalt, T. Schimmel, M. Wegener, K. Okhawa, and D. Hommel, Appl. Phys. Lett. 76, 203 (2000).

${ }^{6}$ G. von Freymann, E. Kurtz, C. Klingshirn, and M. Wegener, Appl. Phys. Lett. 77, 394 (2000).

${ }^{7}$ F. Intonti, V. Emiliani, C. Lienau, T. Elsaesser, V. Savona, E. Runge, R. Zimmermann, R. Nötzel, and K. H. Ploog, Phys. Rev. Lett. 87, 076801 (2001).

${ }^{8}$ C. Adelmann, J. Hetzler, G. Scheiber, Th. Schimmel, M. Wegener, H. B. Weber, and H. v. Löhneysen, Appl. Phys. Lett. 74, 179 (1999).

${ }^{9}$ G. von Freymann, U. Neuberth, M. Deubel, M. Wegener, G. Khitrova, and H. M. Gibbs, Phys. Rev. B (unpublished).

${ }^{10}$ H. F. Hess, E. Betzig, T. D. Harris, L. N. Pfeifer, and K. W. West, Science 264, 822 (1994).

${ }^{11}$ A. Zrenner, L. V. Butov, M. Hagn, G. Abstreiter, G. Böhm, and G. Weimann, Phys. Rev. Lett. 72, 3382 (1994).

${ }^{12}$ U. Bockelmann, W. Heller, A. Filoramo, and P. Roussignol, Phys. Rev. B 55, 4456 (1997).

${ }^{13}$ J. Martinez-Pastor, A. Vinattieri, L. Carraresi, M. Colocci, P. Roussignol, and G. Weimann, Phys. Rev. B 47, 10456 (1993).

${ }^{14}$ S. D. Baranovskii, R. Eichmann, and P. Thomas, Phys. Rev. B 58, 13081 (1998).

${ }^{15}$ E. Runge and R. Zimmermann, Phys. Status Solidi B 221, 269 (2000).

${ }^{16}$ K. Leosson, J. R. Jensen, J. M. Hvam, and W. Langbein, Phys. Status Solidi B 221, 49 (2000). 